УДК 811.112 .2

DOI: https://doi.org/10.28925/2311-2425.2019.12.7

\title{
KONZEPTUELLE UND LEXIKALISCHE METONYMISCHE MODELLE DER ABGELEITETEN SUFFIXALEN SUBSTANTIVE DER MODERNEN DEUTSCHEN SPRACHE
}

\author{
IIchuk O.A., \\ Borys Grinchenko Kyiv University, \\ Bulvarno-Kudriavska Straße 18/2b Kyiv, 04053 \\ o.ilchuk@kubg.edu.ua \\ ORCID iD 0000-0001-9377-1338
}

\begin{abstract}
Der vorgeschlagene Artikel ist dem Vergleich von konzeptuellen und lexikalischen metonymischen Modellen der abgeleiteten suffixalen Substantive des modernen Deutschen mit echten deutschen Suffixen gewidmet. Es wird eine vergleichende Analyse der Typologie konzeptueller und lexikalischer metonymischer Modelle im Allgemeinen sowie am Beispiel einer engeren Gruppe lokaler Metonymietypen durchgeführt.
\end{abstract}

Schlüsselwörter: konzeptuelles Modell, metonymisches Modell, lokale Metonymie, abgeleitete Einheiten, deutsche Sprache.

Ільчук О.A.

\section{Концептуальні та лексичні метонімічні моделі похідних суфіксальних іменників сучасної німецької мови}

Запропонована стаття присвячена зіставленню концептуальних та лексичних метонімічних моделей похідних суфіксальних іменників сучасної німецької мови з власне німецькими суфіксами. здійснено порівняльний аналіз типології концептуальних та лексичних метонімічних моделей загалом, а також на прикладі вужчої групи локального типу метонімії.

Ключові слова: концептуальна модель, метонімічна модель, локальна метонімія, похідні одиниці, німецька мова.

\section{O. Ilchuk}

Conceptual and lexical metonymic models of the derivative suffixal nouns of the modern German language

In linguistic researches of previous years sufficient attention is paid to the study of lexical and conceptual metonymy. In addition, word-formation units may also contain a metonymic component. In the proposed study we aim to compare the typology of conceptual and lexical metonymic models on the material of the suffixal nouns of modern German.

The material of the study is about 3.600 lexical-semantic variants of the derivative suffixal nouns of modern German, selected by the method of a continuous sample of dictionaries. Semantic modeling is involved in constructing semantic models of derivative words, and cognitive modeling serves to construct cognitive models of the studied linguistic units.

The metonymic model in general differs from other models by the presence of a metonymic transfer. In the group of suffixal nouns the participants in the metonymic relation are the deriving and derived units, as well as the suffixal formant. The basis of the conceptual metonymic model is the metonymic relation of the DERIVING UNIT to the DERIVED UNIT with the participation of the suffix. The basis of the lexical metonymic model of derivative units is the word-forming meaning, which is the result of the interaction of the derived unit and the suffix within a certain word-building model. The metonymic models are presented in the studied units by the causal, local, attributive and quantitative types.

As example is a comparison of local metonymy given below. A conceptual metonymic model of the local type "LOCATION instead of ACT" is based on the replacement of the concept of the DERIVING UNIT, which presents the word schmieden, by the concept of the DERIVED UNIT, which represents the word Schmiede, where the suffix -e expresses the substitution of the ACTION to the LOCATION. The corresponding conceptual model is the lexical metonymic model "action - the place of its execution", which is based on the word-building meaning 'where the action is performed, called by the verb' (Schmiede by schmieden).

The typology of conceptual and lexical models of derivative suffixal nouns with genuine German suffixes on an example of a group of local relations is considered in greater detail. The local type of metonymic relation 
between members of a metonymic model is represented in this group of derivative nouns by four conceptual models and the same number of word-forming metonymic models.

The given models of derivative suffixal nouns of modern German coincide by the type of metonymic relation on the lexical-word-building and cognitive levels. This testifies to the similarity of the lexical-word-building and conceptual representation of types of metonymical relations in derivative units.

Key words: conceptual model, metonymic model, local metonymy, derivative units, German.

Einleitung. Die Relevanz dieser Studie wird durch die allgemeine Tendenz der Sprachstudien bestimmt, verschiedene Arten kognitiver und sprachlicher Strukturen $\mathrm{zu}$ untersuchen. Die vorgeschlagene Studie zielt darauf ab, die Typologie konzeptueller und lexikalischer metonymischer Modelle auf dem Material der Suffixalnomen des heutigen Deutschen zu vergleichen.

Eine kritische Überprüfung der Literatur, der konzeptionellen Rahmen, Hypothesen und dergleichen. In den sprachlichen Forschungen der vergangenen Jahre wurde ausreichend Aufmerksamkeit der Untersuchung lexikalischer (O.K. Birich , M.Ya. Beach, V.V. Zaitseva, O. Koroleva, O.P. Nalobina, O. Nikitina, A.L. Novikov, M.S. Sandakova, A.A. Taranenko, A.G. Udinska, O.V. Shelestyuk und andere) und konzeptioneller Metonymie gewidmet (M.M. Boldyrev, O.P. Kapranov, O.Yu. Rezinkin, H.-H. Drößiger, G. Lakoff, Z. Kövecses und K.-U. Panther u.a.). Gleichzeitig können Wortbildungseinheiten auch eine metonymische Komponente enthalten.

Aufgrund der Anwesenheit der metonymischen Komponente in der Struktur der abgeleiteten Substantive scheint der Versuch ihrer Typologie aufgrund des Aufbaus der metonymischen Modelle auf der sprachlichen und konzeptuellen Ebenen möglich. Die Typologie lexikalischer metonymischer Modelle kann auf der Tatsache beruhen, dass wortbildende Einheiten auch Einheiten der lexikalischen Ebene der Sprache sind. Daher sollte man zu ihnen die allgemeine Typologie der lexikalischen Metonymie anwenden, nach der metonymische Übertragungen in kausale, attributive, lokale und u.a. vereint werden. Die Typologie der konzeptuellen metonymischen Modelle kann auf dem Konzept der kognitiven Metonymie von George Lakoff beruhen und von der Tatsache ausgehen, dass die konzeptionelle Struktur der abgeleiteten Substantive eine metonymische Komponente enthält (das Ergebnis des konzeptionellen Ersatzes des Konzepts der ableitenden Einheit durch das Konzept der abgeleiteten Einheit), deshalb scheint ihre Typologie nach der Art der konzeptuellen metonymischen Beziehung rechtmäßig zu sein.

Methodik der Forschung. Als Material der Forschung dienen etwa 3.600 lexikalisch-semantische Varianten der abgeleiteten suffixalen Substantive des modernen Deutsch, die durch kontinuierliche Probenahme aus dem Wörterbuch ausgewählt sind. Ein konzeptionelles metonymisches Modell von abgeleiteten
Substantiven mit dem suffixalen Formant demonstriert die Ersetzung des Konzepts ABLEITENDE EINHEIT durch das Konzept ABGELEITETE EINHEIT mit der Teilnahme des Suffixes, das das Substitutionsverhältnis festlegt. Ein lexikalisches metonymisches Modell der abgeleiteten Substantive mit dem suffixalen Formant spiegelt die Beziehung des metonymischen Typs zwischen dem motivierenden Wort und dem motivierten Nomen wider. Semantische Modellierung ist beim Aufbau semantischer Modelle abgeleiteter Wörter verwendet. Die kognitive Modellierung diente dazu, kognitive Modelle der untersuchten Spracheinheiten zu konstruieren.

Forschung und Diskussion. Das metonymische Modell unterscheidet sich im Allgemeinen von anderen Modellen durch das Vorhanden der metonymischen Übertragung. In der Gruppe der suffixalen Substantive sind die Teilnehmer der metonymischen Übertragung eine ableitende Einheit und eine abgeleitete Einheit sowie der suffixale Formant. Die Basis des konzeptuellen metonymischen Modells ist die metonymische Beziehung der ABLEITENDEN EINHEIT zur ABGELEITETEN EINHEIT unter Beteiligung des Suffixes. Die Basis des lexikalischen metonymischen Modells der abgeleiteten Einheiten ist die wortbildende Bedeutung, die das Ergebnis der Interaktion der ableitenden Einheit und des Suffixes innerhalb eines bestimmten Wortbildungsmodells ist. Die metonymischen Modelle werden in den untersuchten Einheiten nach kausalem, lokalem, attributivem und quantitativem Typ dargestellt.

Konzeptuelles metonymisches Modell des kausalen Typs "ERGEBNIS DER HANDLUNG statt HANDLUNG" basiert auf der Ersetzung des Konzepts ABLEITENDE EINHEIT, das das Wort kühlen darstellt, durch das Konzept ABGELEITETE EINHEIT, das das Wort Kühlung repräsentiert, wo Suffix - ung die Ersetzung einer HANDLUNG durch das ERGEBNIS DER HANDLUNG ausdrückt. Korrespondierend mit diesem konzeptionellen Modell ist das lexikalische metonymische Modell "Handlung $\rightarrow$ Ergebnis der Handlung", das auf der wortbildenden Bedeutung 'Ergebnis nach der durch das Verb benannten Handlung' (Kühlung von kühlen) basiert. Ein Beispiel für die Implementierung dieses metonymischen Modells ist die Aussage "Grünflächen absorbieren Wasser und sorgen durch schuldig Luft für Kühlung [Die Zeit, 24. November 2015]".

Konzeptuelles metonymisches Modell des lokalen Typs "ORT statt HANDLUNG" basiert 
auf der Ersetzung des Konzepts ABLEITENDE EINHEIT, das das Wort schmieden präsentiert, durch das Konzept ABGELEITETE EINHEIT, das das Wort Schmiede präsentiert, wo Suffix -e den Ersatz der HANDLUNG zum ORT ausdrückt. Relevant diesem metonymischen Modell ist das lexikalische metonymische Modell "Handlung $\rightarrow$ Ort ihrer Ausführung", das auf der wortbildenden Bedeutung basiert, 'wo die Aktion ausgeführt wird', das als Verb bezeichnet wird' (Schmiede von schmieden). Als Beispiel für dieses metonymische Modell geben wir folgende Aussage an: "Nun sollen sterben RenaultMotoren wohl zusätzlich in der Schmiede von Mario Illien weiterentwickelt werden [Die Zeit, 27.11.2015]".

Konzeptuelles metonymisches Modell des attributiven Typs "PERSON statt MERKMAL" basiert auf der Ersetzung des Konzepts ABLEITENDE EINHEIT, das das Wort ein darstellt, durch das Konzept ABGELEITETE EINHEIT, das das Wort Einheit darstellt, wo Suffix -heit den Ersatz MERKMAL zur PERSON ausdrückt. Ähnlich diesem konzeptuellen Modell ist das lexikalische metonymische Modell „Merkmal $\rightarrow$ die Person, die sie besitzt", die auf der wortbildenden Bedeutung 'besitzt das Merkmal, genannt vom Kardinalzahl' (Einheit von ein) basiert. Ein Beispiel für die Verwendung dieses metonymischen Modells kann die Aussage sein "Unsere Einheiten haben es geschafft, in das Regierungsviertel vorzurücken", sagte ein Polizeisprecher am Sonntag der Deutschen PresseAgentur [Die Zeit, 27.12.2015].

Konzeptuelles metonymisches Modell des quantitativen Typs "GESAMTHEIT VON PERSONEN statt PERSON" basiert auf der Ersetzung des Konzepts ABLEITENDE EINHEIT, das das Wort Hörer darstellt, durch Konzept ABGELEITETE EINHEIT, das das Wort Hörerschaft präsentiert, wo Suffix -schaft die Ersetzung von PERSON durch GESAMTHEIT VON PERSONEN ausdrückt. Messbar zu diesem konzeptionellen Modell ist das lexikalische metonymische Modell "Person $\rightarrow$ eine Gesamtheit von Personen", basierend auf der wortbildenden Bedeutung 'eine Gruppe von Personen’ (Hörerschaft von Hörer). Als Beispiel für dieses metonymische Modell zitieren wir "Man muss nicht mehr versuchen, eine noch unbekannte Band bei einer Hörerschaft durchzusetzen, von der man nicht weiß, was diese eigentlich vorgesetzt bekommen möchte [Die Zeit, 27.01.2015]".

Weiter betrachten wir die Typologie der konzeptuellen und lexikalischen Modelle der abgeleiteten suffixalen Substantive mit echten deutschen Suffixen am Beispiel einer Gruppe der lokalen Beziehung. Der lokale Typ der metonymischen Beziehung zwischen den Mitgliedern des metonymischen Modells ist in dieser Gruppe der abgeleiteten Substantive mit vier konzeptuellen Modellen und der gleichen Zahl der lexikalischen metonymischen Modelle dargestellt:
1. Modell №1. Das konzeptuelle Modell “ORT statt HANDLUNG":

Norddeutschland ist zum Beispiel sehr flach, es gibt weniger Abwechslung wie Berge, Wäldern, Wiesen, Weiden, Hecken [Die Zeit, 25.03.2016]. Das lexikalische Modell "Handlung $\rightarrow$ Ort ihrer Ausführung": Zudem soll Brot künftig außer in Bäckereien und Supermärkten auch in anderen Lebensmittelläden verkauft werden können [Die Zeit, 31.03.2014].

2. Modell №2. Das konzeptuelle Modell "PERSON statt ORT": Die Osteuropäer haben die Sorge, dass neben Großbritannien künftig auch andere EU-Staaten - beispielsweise Deutschland - von der „Notbremse" Gebrauch machen [Der Tagesspiegel, 18.02.2016]. Das lexikalische Modell "Ort $\rightarrow$ die Person, die da wohnt, ist, arbeitet oder studiert": Tausende Neuseeländer haben Prinz William und Ehefrau Kate einen begeisterten Empfang bereitet [Die SZ, 10.04.2014].

3. Modell №3. Das konzeptuelle Modell "ORT statt PERSON": Jetzt redet ein gedrungener Mann mit Zylinderhut auf sie ein: Hendrik Haase, der im Herbst unter deutschlandweiter Anteilnahme eine Metzgerei in Driessens Halle eröffnet hat [Der Tagesspiegel, 12.04.2016]. Das lexikalische Modell "Person $\rightarrow$ Ort, wo sie da wohnt, ist, arbeitet oder studiert місце": Wir warten gespannt auf die Eröffnung der Konditorei Zucker und der Imbissstube Fett [Die Zeit, 11.06.2015].

4. Modell №4. Das konzeptuelle Modell “ORT statt OBJEKT": Bei GNTM hat 2015 die Schweizer Molkerei Emmi diese Möglichkeit genutzt und ein Getränk nachträglich in die Sendung montieren lassen [Die Zeit, 04.02.2016]. Das lexikalische Modell "Gegenstand $\rightarrow$ Ort der Handlung damit": Angestellte der Fischerei erkannten ihren alte Kollegen jetzt auf den Pressefotos wieder [Die SZ, 06.02.2014].

Die gegebenen Modelle der abgeleiteten suffixalen Substantive des modernen Deutschen fallen durch die Art der metonymischen Beziehung auf der lexikalisch-wortbildenden und der kognitiven Ebene zusammen. Das zeigt dieselbe lexikalischwortbildende Wiederspiegelung der metonymischen Beziehung in den abgeleiteten Einheiten der erforschten Gruppe.

Schlussfolgerungen und Perspektiven weiterer Forschung. In dem Artikel wurde eine vergleichende Analyse der Typologie konzeptueller und lexikalischer metonymischer Modelle der abgeleiteten suffixalen Substantive der modernen deutschen Sprache durchgeführt. Im Allgemeinen wurde festgestellt, dass typologisch gesehen die metonymische Komponente gleichermaßen auf der kognitiven und sprachlichen Ebenen realisiert wird. Perspektivische Richtungen der weiteren Forschung in der modernen Germanistik können das Studium der metonymischen Komponente anderer Wortarten und die Beschreibung der kognitiven Metonymie der abgeleiteten Substantive in verschiedenen Diskursarten sein. 


\section{REFERENCES}

1. Birikh, A. (1995). Metonimiia v sovremennom russkom yazyke: semanticheskii i grammaticheskii aspekty. München: Verlag Otto Sagner, 195 p.

2. Bich, M. (1995). Ya. Metonimicheskoe ispolzovanie imion sobstvennykh v sovremennom russkom, ispanskom i angliiskom yazykakh (na materiale gazetnykh tekstov): avtoref. dis. na soiskanie uchen. stepeni kand. filol. nauk: spets. 10.02.19, «Obshchee yazykoznanie, sotsiolingvistika, psikholingvistika», Spb., 18 p.

3. Boldyrev, N. N. (2002). Kontseptualnaia metonimiia na raznykh urovniakh yazyka: sistema i realizatsiia. Forma, znachenie i funktsii yedinits yazyka i rechi: Mat-ly dokl. Mezhdunar. nauch. konf. Minsk: MGLU, Ch. 1, pp. 11-14.

4. Zaitseva, V. V. (2009). Metonimiia yak sposib vtorynnoi nominatsii u movi drukovanykh zasobiv informatsii: avtoref. dys. na zdobuttia nauk. stupenia kand. filol. nauk: spets. 10.02.01. «Ukrainska mova». Dnipropetrovsk, $22 \mathrm{p}$.

5. Kapranov, O. P. (2002). Ideoetnichnyi kharakter kohnitivnoi slovotvirnoi metonimii (na materiali pokhidnykh imennikiv suchasnoi nimetskoi movy). Visnyk Zaporizkogo derzhavnoho universitetu: zbirnyk naukovykh statei, Holovnyi redaktor Tolok V. O., Zaporizhzhia: Zaporizkyi derzhavnii universitet Filolohichni nauky, № 1, pp. 51-60.

6. Koroleva, O. E. (2003). Problemy substantivnoi metonimii: avtoreferat dis.... kandidata filologicheskikh nauk: 10.02 .01 , Kaluga, $16 \mathrm{p}$.

7. Nalobina, Ye. P. (2014). Osobennosti obrazovaniia proizvodnykh znachenii deadektivnykh sushchestvitelnykh $\mathrm{v}$ nemetskom yazyke (na osnove metonimicheskogo perenosa). Novosibirsk: Izd-vo NGPU, Aktualnyie problemy filologii i metodiki prepodavaniia inostrannykh yazykov, 8, pp. 49-55.

8. Nikitina, O. A. (2010). Osobennosti obrazovaniia proizvodnykh znachenii slov na osnove metonimicheskogo perenosa $\mathrm{v}$ sovremennom nemetskom yazyke (na materiale neologizmov kontsa XX - nachala XXI vv.). Cheliabinsk: Izd-vo: ChGPU, Vestnik Cheliabinskogo Gosudarstvennogo Pedagogicheskogo Universiteta, 4, pp. 256-271.

9. Rezinkin, A. Yu. (2011). Kognitivnyie podkhody k izucheniiu metonimii. MNKO, № 6-2, pp. 32-35.

10. Sandakova, M. V. (2004). Metonimiia prilagatelnogo v russkom yazyke: avtoreferat dis. ... doktora filologicheskikh nauk: 10.02.01. Mosk. ped. gos. un-t., Moskva, $36 \mathrm{p}$.

11. Taranenko, O. O. (2007). Metonimiia. Ukrainska mova: Entsyklopediia, 3-tie vyd., K.: Ukr. entsyklopediia, pp. 312-314.

12. Udinska, A. H. (2007). Metonimichni perenosy kauzalnoho typu na poznachennia liudyny v anhliiskii ta ukrainskii movakh: avtoref. dys. na zdobuttia nauk. stupenia kand. filol. nauk: spetsc. 10.02.17. «Porivnialno-istorychne i typolohichne movoznavstvo». Donetsk, 19 p.

13. Shelestiuk, Ye. V. (2002). Metonimiia kak sposob semanticheskoi derivatsii (o tipakh metonimicheskikh konstruktsii v semanticheskoi strukture angliiskikh sushchestvitelnykh). Aktualnyie problemy yazykoznaniia i metodiki obucheniia inostrannym yazykam, Cheliabinsk: Izd-vo ChGPU, pp. 38-40.

14. Busse, D. (2009). Semantik. Paderborn: Fink, 144 p.

15. Lakoff, G. \& Johnson, M. (2003). Metaphors We Live by. The University of Chicago Press, Chicago.

16. Motsch, W. (2004). Deutsche Wortbildung in Grundzügen / Wolfgang Motsch. [2. Aufl.]. Berlin, N. Y.: W. de Gruyter, 458 S.

17. Radden, G. \& Kovesces, Z. (1999). Towards a Theory of Metonymy. K. Panther, G. Radden (Eds.). Metonymy in Language and Thought, Amsterdam, Philadelphia: John Benjamins, pp. 17-61.

18. Süddeutsche Zeitung Online.

https://www.sueddeutsche.de/

19. Tagesspiegel Online.

https://www.tagesspiegel.de/

20. Zeit Online.

http://www.zeit.de

Дата надходження статті до редакції: 18.03.2019.

Прийнято до друку: 16.04.2019. 